\title{
Fatores sociodemográficos e imagem corporal em adolescentes do ensino médio
}

\author{
Sociodemographic factors and body image \\ among high school students
}

Érico Pereira Gomes Felden ${ }^{1}$

Gaia Salvador Claumann ${ }^{1}$

Cinara Sacomori ${ }^{1}$

Luciane Sanchotene Etchepare Daronco ${ }^{2}$

Fernando Luiz Cardoso ${ }^{1}$

Andreia Pelegrini ${ }^{1}$

${ }^{1}$ Centro de Ciências da Saúde e do Esporte, Universidade do Estado de Santa Catarina. R. Pascoal Simone 358, Coqueiros. 88080-350 Florianópolis SC Brasil.

ericofelden@gmail.com

${ }^{2}$ Departamento de

Desportos Coletivos,

Centro de Educação Física

e Desportos, Universidade

Federal de Santa Maria.
Abstract The scope of this article is to investigate associations between body image dissatisfaction and sociodemographic indicators among adolescents. Participants included 1126 high school students in the city of Santa Maria in the State of Rio Grande do Sul. Aspects regarding body image dissatisfaction and sociodemographic indicators were analyzed. Association analyses were performed using crude Poisson regression duly adjusted for sex and age. Boys were found to be dissatisfied due to slimness and girls due to excess weight. Adolescents where the parents had less schooling, as well as those from lower socioeconomic classes and with lower income were found to be more likely to have body image dissatisfaction due to slimness. However, those where the parents had completed higher education and those living in the downtown area were more likely to have body image dissatisfaction due to excess weight. Sociodemographic indicators are closely associated with body image dissatisfaction. Body image dissatisfaction due to slimness can be explained by the lower level of schooling of the parents, lower socioeconomic class and lower income. Dissatisfaction due to excess weight can be explained by the higher schooling of the parents and the fact of living in the downtown area.

Key words Body image, Social class, Economic indicators, Demographic indicators
Resumo O objetivo deste artigo é investigar associações entre a insatisfação com a imagem corporal e indicadores sociodemográficos em adolescentes. Participaram 1126 adolescentes do ensino médio do município de Santa Maria/RS. Foram analisadas questões sobre a insatisfação com a imagem corporal e indicadores sociodemográficos. As análises de associação foram realizadas por meio de regressão de Poisson bruta e ajustada por sexo e faixa etária. Rapazes estão insatisfeitos pela magreza e moças pelo excesso de peso. Os adolescentes cujo chefe de família possui menor escolaridade, de classes econômicas mais baixas e com menor renda apresentam maior probabilidade de insatisfação pela magreza. Já aqueles com o chefe de família apresentando ensino superior completo e os que residem no centro da cidade têm maior probabilidade de insatisfação pelo excesso de peso. Indicadores sociodemográficos estão associados à insatisfação com a imagem corporal. A insatisfação pela magreza é explicada pela menor escolaridade do chefe de família, pertencer às classes econômicas mais populares e ter menor renda. Já a insatisfação pelo excesso de peso, pela maior escolaridade do chefe de familia e residir no centro. Palavras-chave Imagem corporal, Classe social, Indicadores econômicos, Indicadores demográficos 


\section{Introdução}

Inúmeros fatores influenciam na forma como as pessoas percebem o próprio corpo e em como se sentem em relação a essa percepção ${ }^{1}$. Primordialmente, é esperado que um sistema nervoso central maduro e desenvolvido de forma adequada consiga perceber com qualidade as dimensões corporais e onde o corpo e os membros estão no espaço ${ }^{2,3}$. Assim, a forma como são percebidas as dimensões e o formato do corpo e a imagem formada na mente a partir desta percepção deve ser analisada de forma multifatorial, considerando, além dos aspectos físicos/motores, os sociodemográficos, culturais e históricos ${ }^{4-7}$.

A imagem corporal é especialmente associada com variáveis neurofisiológicas e antropométricas. Apesar disso, evidências apontam para a importância dos determinantes sociais nos sentimentos de satisfação/insatisfação com o corpo, especialmente na adolescência, sugerindo, desta forma, que as influências culturais e de padrões de beleza podem ser constituídas de formas singulares de acordo, por exemplo, com a classe socioeconômica ${ }^{8}$.

O estudo de Pereira et al. ${ }^{6}$, conduzido em crianças e adolescentes do município de Florianópolis/SC, é um dos pioneiros no Brasil a demonstrar que jovens de diferentes classes econômicas podem possuir sentimentos distintos em relação ao seu corpo. Neste estudo foi verificado, por exemplo, que os jovens da classe baixa apresentaram maior insatisfação pelo excesso de peso $^{6}$. Já Dumith et al. ${ }^{9}$, em investigação conduzida com adolescentes de Pelotas/RS, verificaram maior proporção de meninos da classe alta insatisfeitos com o excesso de peso em relação aos seus separes das demais classes econômicas, enquanto houve maior proporção de rapazes e moças da classe baixa insatisfeitos pela magreza. Por sua vez, entre crianças e adolescentes de Cascavel/PR e Caxias do Sul/RS, Fidelix et al. ${ }^{10}$ e Finato et al. ${ }^{11}$, respectivamente, não identificaram diferenças nas prevalências de insatisfação com a imagem corporal nas classes investigadas.

Neste contexto, destacam-se algumas questões como as altas prevalências de insatisfação com o corpo, especialmente nos adolescentes, observadas nos estudos; as limitações da literatura no que se refere às associações da imagem corporal com diferentes indicadores socioeconômicos; e ainda, a utilização de diferentes parâmetros sociais nos estudos, o que limita o conhecimento das tendências de comportamento bem como a comparação com estudos internacionais.
Assim, este estudo se propõe a avançar em alguns pontos em relação aos publicados sobre imagem corporal investigando as associações entre a insatisfação com a imagem corporal e diferentes indicadores sociodemográficos, como classe econômica, renda, local de moradia e cor da pele.

\section{Método}

\section{Amostra}

Analisaram-se 1126 adolescentes (506 rapazes e 620 moças), de 13 a 21 anos de idade, estudantes do ensino médio, matriculados na rede pública de ensino, no ano de 2009, no município de Santa Maria/RS, Brasil. Santa Maria é considerado um dos principais municípios do estado do Rio Grande do Sul. Seu Índice de Desenvolvimento Humano (IDH) no ano de 2000 foi de 0,845 , o que indica ser uma região de alto desenvolvimento humano. O projeto de pesquisa foi aprovado pelo Comitê de Ética em Pesquisa com Seres Humanos da Universidade Federal do Paraná, no ano de 2009.

O tamanho da amostra foi calculado considerando-se um erro amostral de três pontos percentuais $^{12}$. Utilizou-se um efeito de delineamento de 1,5 e um percentual de $15 \%$ para compensar possíveis perdas e recusas. A amostra foi estratificada considerando-se as regiões geográficas do município e as zonas urbana e rural.

As escolas sorteadas foram informadas sobre os objetivos da pesquisa e os procedimentos que seriam realizados. Após a autorização da direção da escola, selecionaram-se as turmas de forma aleatória. Os alunos foram informados sobre o estudo e todos, em sala de aula, convidados a participar. Aqueles que concordaram em participar receberam o "Termo de Consentimento Livre e Esclarecido" contendo informações detalhadas da pesquisa.

\section{Critérios de inclusão e exclusão}

Todos os adolescentes matriculados nas escolas foram elegíveis ao estudo. Participaram aqueles que estavam presentes na aula no momento das coletas, que apresentaram o Termo de Consentimento Livre e Esclarecido assinado pelos responsáveis (adolescentes menores de 18 anos de idade) e que aceitaram e responderam adequadamente aos questionários. Foram excluídas as alunas gestantes. 
da, preta).

b) imagem corporal: analisou-se a insatisfação com a imagem corporal por meio da escala de silhuetas, proposta por Stunkard et al. ${ }^{13}$, que consiste num conjunto de nove figuras do sexo masculino e feminino, com variações progressivas na escala de medida. A aplicação desenvolveu-se a partir da percepção individual, solicitando-se ao sujeito escolher o número da silhueta que considerou semelhante a sua aparência corporal real (Imagem Corporal Real - ICR) e, também, com sua aparência corporal ideal (Imagem Corporal Ideal - ICI). Para a avaliação da insatisfação corporal subtraiu-se da aparência corporal real a aparência corporal ideal, podendo, este índice, variar de menos oito até oito. Obtendo-se uma variação igual a zero, o indivíduo foi classificado como satisfeito com sua aparência e, se diferente de zero, como insatisfeito. Caso a diferença tenha sido positiva considerou-se insatisfação pelo excesso de peso e, quando negativa, insatisfação pela magreza.

c) nível econômico e renda: o nível econômico foi avaliado por meio do questionário da Associação Brasileira de Empresas de Pesquisa ${ }^{14}$, que visa estimar o poder de compra das pessoas e famílias e escolaridade do chefe de família, classificando os indivíduos em estratos econômicos (A1, A2, B1, B2, C, D, E). No presente estudo, os estratos foram agrupados e considerados classe econômica baixa (D e E), média (C) e alta (A1, A2, B1 e B2). Os adolescentes também deveriam indicar sua renda familiar em salários mínimos, de acordo com o valor vigente à época da coleta de dados. Para fins de análise a renda foi agrupada em 1 a 3 salários mínimos, de 3,01 a 6 e, acima de 6 salários mínimos.

\section{Análises estatísticas}

$\mathrm{Na}$ análise descritiva das variáveis, identificaram-se os valores de tendência central (média) e de dispersão (desvio padrão). Os testes de Kruskal-Wallis e Qui-quadrado foram utilizados para identificar diferenças entre os grupos de acordo com o tipo de variável. As associações entre imagem corporal e os indicadores sociode- mográficos foram analisadas por meio do cálculo de razões de prevalências (RP) utilizando-se da regressão de Poisson considerando desfechos com elevadas prevalências ${ }^{15}$. Foram apresentadas análises brutas e ajustadas por sexo e idade. Em todas as análises foi fixado nível de significância de 5\%.

\section{Resultados}

Os dados descritivos da amostra estão apresentados na Tabela 1. A média de idade foi de 16 anos. Mais de $90 \%$ dos adolescentes foram classificados nas classes econômicas média e alta, considerando o poder de compra e a escolaridade do chefe de família. No entanto, apenas 15,5\% da amostra possuía renda familiar de mais de seis salários mínimos vigentes no momento da coleta. Houve diferença na proporção de trabalhadores entre os sexos sendo que este grupo foi formado em sua maioria pelos rapazes $(\mathrm{p}<0,001)$.

Ainda com relação às variáveis que caracterizam a amostra, não foram identificadas diferenças de proporção na variável cor da pele entre os sexos $(p=0,088)$. Apesar disso, foi verificada associação entre cor da pele e classe econômica $(\mathrm{p}=0,016)$ com maior percentual de brancos e pardos na classe alta. Este resultado foi semelhante ao observado com a variável renda $(\mathrm{p}=0,011)$. Não foi identificada associação entre cor da pele e escolaridade do chefe de família $(\mathrm{p}=0,717)$.

A análise da insatisfação com a imagem corporal entre os sexos identificou que o grupo de insatisfeitos pela magreza foi formado, em sua maioria, por rapazes $(\mathrm{p}<0,001)$. Já o grupo de insatisfeitos pelo excesso de peso apresentou maior frequência de moças $(\mathrm{p}<0,001)$. Importante destacar que embora a maioria dos rapazes esteja insatisfeita pela magreza, um percentual expressivo deste grupo apresentou insatisfação pelo excesso de peso $(31,6 \%)$. O percentual de adolescentes satisfeitos com sua imagem corporal foi semelhante entre os sexos $(\mathrm{p}=0,974)$ (Figura 1).

A análise de associação identificou que os rapazes $(\mathrm{RP}=2,19$, IC95\% $=1,72-2,80)$ possuem maior prevalência de insatisfação com a magreza mesmo após ajuste por faixa etária. Com relação aos fatores sociodemográficos, identificou-se que aqueles adolescentes cujo chefe de família possui menor escolaridade (analfabeto/3a série fundamental: $\mathrm{RP}=1,88$, IC95\% $=1,20-2,97$; até $4^{\text {a }}$ série fundamental: RP $=1,67$, IC95\% $=1,10$ 2,56; fundamental completo: RP $=1,68$, IC95\% =1,11-2,54) apresentam maior insatisfação com 


\begin{tabular}{|c|c|c|c|c|}
\hline 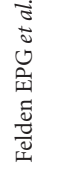 & Tabela 1. Dados descritivos da amos & & & \\
\hline & & & & \\
\hline & Variáveis & Moças $=620$ & Rapazes $=526$ & p-valor \\
\hline & Idade, anos, $\overline{\mathrm{x}}(\mathrm{dp})$ & $16,2(1,4)$ & $16,3(1,4)$ & 0,055 \\
\hline & Faixas etárias, anos, \% & & & 0,119 \\
\hline & $13-14$ & 10,2 & 7,7 & \\
\hline & $15-16$ & 51,6 & 50,2 & \\
\hline & $17-18$ & 33,8 & 35,0 & \\
\hline & $19-21$ & 4,4 & 7,1 & \\
\hline & Classe econômica, \% & & & 0,178 \\
\hline & Baixa & 6,2 & 4,8 & \\
\hline & Média & 44,2 & 40,2 & \\
\hline & Alta & 49,6 & 55,0 & \\
\hline & Renda, \% & & & $<0,001$ \\
\hline & 1-3 salários mínimos & 62,0 & 50,3 & \\
\hline & 3,01-6 salários mínimos & 22,5 & 30,3 & \\
\hline & $>6$ salários mínimos & 15,5 & 19,4 & \\
\hline & Escolaridade do chefe de família, \% & & & 0,133 \\
\hline & Analfabeto/3a Série Fundamental & 14,5 & 11,5 & \\
\hline & Até 4a Série Fundamental & 21,5 & 18,9 & \\
\hline & Fundamental completo & 18,0 & 22,3 & \\
\hline & Médio completo & 27,5 & 25,4 & \\
\hline & Superior completo & 18,5 & 21,9 & \\
\hline & Cor da pele, $\%$ & & & 0,088 \\
\hline & Branca & 71,2 & 65,6 & \\
\hline & Parda & 19,6 & 21,6 & \\
\hline & Preta & 9,2 & 12,8 & \\
\hline & Local de moradia, $\%$ & & & 0,579 \\
\hline & Periferia & 86,2 & 87,4 & \\
\hline & Centro & 11,6 & 9,9 & \\
\hline & Rural & 2,2 & 2,7 & \\
\hline & Trabalhadores, \% & 18,9 & 31,2 & $<0,001$ \\
\hline & Carga de trabalho semanal & & & 0,413 \\
\hline & Até 20 horas & 17,1 & 13,4 & \\
\hline & Mais que 20 horas & 82,9 & 86,6 & \\
\hline & Turno escolar, \% & & & 0,196 \\
\hline & Matutino & 55,6 & 52,4 & \\
\hline & Vespertino & 26,0 & 24,9 & \\
\hline & Noturno & 18,4 & 22,7 & \\
\hline
\end{tabular}

$\bar{x}$ : média; dp: desvio padrão; \%: frequência relativa.

a magreza na análise ajustada por sexo e faixa etária. Também na análise ajustada identificouse maior insatisfação com a magreza na classe econômica baixa $(\mathrm{RP}=1,98$, IC95 $=1,29-3,04)$ e nos adolescentes com menor renda $(\mathrm{RP}=1,46$, IC95\% = 1,02-2,09) (Tabela 2).

Em relação à insatisfação pelo excesso de peso, observou-se que a probabilidade de apresentar o desfecho foi maior entre as moças (RP $=1,65$, IC95\% = 1,36-2,01). Além disso, verificou-se que os adolescentes cujo chefe de família possui maior grau de escolaridade $(\mathrm{RP}=1,38$, IC95\% $=1,08-1,77)$ e aqueles que residem no centro $(\mathrm{RP}=2,43$, IC95\% = 1,10-5,35) apresentaram probabilidade maior de estarem insatisfeitos pelo excesso (Tabela 3).

\section{Discussão}

Não obstante ao que a literatura ${ }^{6,10,16,17}$ relata sobre a elevada proporção de adolescentes insatisfeitos com a imagem corporal, o presente estudo também encontrou prevalência preocupante desse desfecho nos adolescentes participantes (69,7\%, dado não apresentado). Uma das possí- 


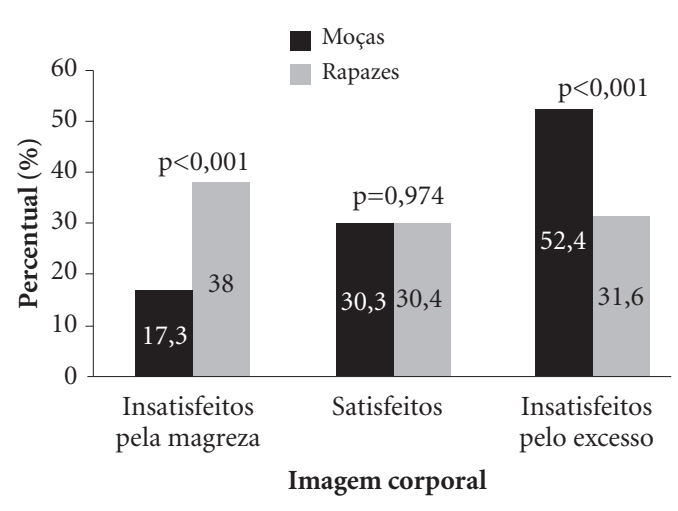

Figura 1. Satisfação com a imagem corporal estratificado por sexo.

veis justificativas para a elevada insatisfação com a imagem corporal pode estar atrelada à facilidade de acesso aos meios midiáticos, principalmente aqueles relacionados às redes sociais, sendo a internet representada como um potente meio sociocultural que contribui para a distorção da imagem corporal ${ }^{18,19}$, ao qual os adolescentes estão frequentemente expostos, tornando-se um grupo de risco.

Atrelados a esse contexto, a idealização e/ou internalização de um corpo perfeito vem sendo incorporada nas sociedades atuais, sendo a beleza apontada como sinônimo de corpo bonito ${ }^{20}$, magro e musculoso ${ }^{4}$. Portanto, o conhecimento da dinâmica da adolescência em relação ao seu corpo, as influências externas e as características associadas são fundamentais para o reconhecimento precoce e a adoção de medidas preventivas de distúrbios da imagem corporal ${ }^{21}$.

Os achados do presente estudo revelam que os rapazes estão mais insatisfeitos pela magreza, enquanto as moças apresentam maior insatisfação pelo excesso de peso. Passos et al. ${ }^{20}$ apontam que há uma busca incessante de um corpo definido e musculoso no sexo masculino, e de corpo magro e curvilíneo no feminino. Tais sentimentos e desejos podem levar o adolescente a adotar comportamentos não saudáveis, principalmente com dietas restritivas de emagrecimento, o que leva a graves distúrbios nutricionais, como a anorexia nervosa e a bulimia nervosa ${ }^{22}$, e práticas excessivas de exercícios físicos e uso de anabolizantes que predispõem aos quadros de dismorfia muscular ${ }^{23}$. Corroborando esses achados, estudos conduzidos no Brasil apontam que a principal razão para a prática de musculação e para o consumo de anabolizantes é a motivação estética ${ }^{24,25}$.

Os adolescentes cujo chefe de família possui menor escolaridade, aqueles das classes econômicas mais baixas e com menor renda estiveram mais suscetíveis à insatisfação pela magreza. Neste contexto, supõe-se que ter um corpo magro ou com pouco volume muscular pode caracterizar um corpo pouco hábil ou útil para a realização de tarefas braçais, mais comuns nos campos de trabalho das camadas populares. Esta hipótese parece ser confirmada por um estudo de revisão sistemática, publicado em 2011, que verificou que os adolescentes de menor nível econômico apresentam maior tendência a desejarem corpos maiores do que os seus atuais ${ }^{8}$.

Em relação à insatisfação pelo excesso de peso, verificou-se que o local de moradia esteve associado ao desfecho. Os adolescentes que residem no centro apresentaram maior probabilidade de desejarem um corpo mais magro. De modo semelhante, Triches e Giugliani ${ }^{26}$ encontraram diferenças na insatisfação corporal de escolares das áreas urbana e rural. Pode-se supor, especialmente no caso do presente estudo, em que o centro e a periferia foram considerados separadamente, ao invés de agrupados em área urbana, que os adolescentes que residem no centro estão mais próximos das publicidades das zonas comerciais e, portanto, mais expostos aos ideais de beleza e magreza que estas frequentemente exibem.

Assim, os jovens da periferia e da área rural poderiam, de certa forma, estar protegidos contra as pressões midiáticas, o que também foi questionado pelas autoras Triches e Giugliani ${ }^{26} \mathrm{em}$ seu estudo. Ainda, pode-se imaginar que existem diferentes padrões de beleza vigentes nestas áreas. Vindo ao encontro desta discussão, tem sido apresentado que a magreza pode ser considerada um marcador social nas sociedades industrializadas $^{27}$, que pode situar ou valorar os indivíduos em uma hierarquia social. Sociólogos ilustram as diferenças de classes socioeconômicas como diferentes formas de investimento e controle do corpo como um projeto de ascensão social ${ }^{28}$, isto explicaria as diferentes estratégias individuais e coletivas de se aspirar, investir e preferir um tipo de corpo adequado para se obter sucesso social em seu meio.

Esta situação pode ser acomodada nas discussões de Bourdieu sobre estilos de vida, em que sugere que os comportamentos são guiados por gostos que se desenvolvem ao longo da vida em função dos recursos financeiros, ocupações pro- 
Tabela 2. Prevalências e razões de prevalências utilizando-se como variável dependente a insatisfação pela magreza.

\begin{tabular}{|c|c|c|c|}
\hline Variáveis & $\%$ & $\begin{array}{l}\text { RP Bruta } \\
\text { (IC 95\%) }\end{array}$ & $\begin{array}{l}\text { RP Ajustada } \\
\text { (IC 95\%) }\end{array}$ \\
\hline \multicolumn{4}{|l|}{ Sexo } \\
\hline Moças & 17,3 & 1,00 & 1 \\
\hline Rapazes & 38,0 & $2,20(1,78-2,72)$ & $2,19(1,72-2,80)$ \\
\hline \multicolumn{4}{|l|}{ Faixas etárias, anos } \\
\hline $13-14$ & 24,0 & 1,00 & 1,00 \\
\hline $15-16$ & 25,6 & $1,07(0,73-1,57)$ & $1,02(0,66-1,59)$ \\
\hline $17-18$ & 27,5 & $1,15(0,77-1,70)$ & $1,09(0,69-1,72)$ \\
\hline $19-21$ & 31,7 & $1,32(0,79-2,21)$ & $1,16(0,63-2,14)$ \\
\hline \multicolumn{4}{|l|}{ Classe econômica } \\
\hline Baixa & 43,1 & $1,84(1,32-2,57)$ & $1,98(1,29-3,04)$ \\
\hline Média & 27,9 & $1,19(0,97-1,48)$ & $1,25(0,97-1,60)$ \\
\hline Alta & 23,4 & 1,00 & 1,00 \\
\hline \multicolumn{4}{|l|}{ Renda } \\
\hline 1-3 salários mínimos & 28,0 & $1,35(0,98-1,84)$ & $1,46(1,02-2,09)$ \\
\hline 3,01-6 salários mínimos & 25,3 & $1,22(0,85-1,73)$ & $1,20(0,81-1,80)$ \\
\hline > 6 salários mínimos & 20,8 & 1,00 & 1,00 \\
\hline \multicolumn{4}{|l|}{ Chefe da família } \\
\hline Analfabeto/3a Série Fund. & 30,1 & $1,71(1,15-2,54)$ & $1,88(1,20-2,97)$ \\
\hline Até 4a Série Fundamental & 27,5 & $1,56(1,08-2,27)$ & $1,67(1,10-2,56)$ \\
\hline Fundamental completo & 29,9 & $1,70(1,18-2,45)$ & $1,68(1,11-2,54)$ \\
\hline Médio completo & 23,6 & $1,34(0,93-1,94)$ & $1,43(0,95-2,17)$ \\
\hline Superior completo & 17,6 & 1,00 & 1,00 \\
\hline \multicolumn{4}{|l|}{ Cor da pele } \\
\hline Branca & 25,7 & 1,00 & 1,00 \\
\hline Parda & 28,8 & $1,12(0,81-1,54)$ & $1,01(0,70-1,47)$ \\
\hline Preta & 26,1 & $1,02(0,78-1,32)$ & $0,97(0,72-1,32)$ \\
\hline \multicolumn{4}{|l|}{ Local de moradia } \\
\hline Periferia & 26,9 & $1,40(0,63-3,10)$ & $1,46(0,60-3,55)$ \\
\hline Centro & 25,2 & $1,31(0,56-3,07)$ & $1,45(0,60-3,78)$ \\
\hline Rural & 19,2 & 1,00 & 1,00 \\
\hline \multicolumn{4}{|l|}{ Trabalhadores } \\
\hline Não & 25,3 & 1,00 & 1,00 \\
\hline Sim & 30,5 & $1,21(0,97-1,50)$ & $1,05(0,80-1,40)$ \\
\hline \multicolumn{4}{|l|}{ Carga de trabalho semanal } \\
\hline Até 20 horas & 37,8 & $1,26(0,79-2,01)$ & $1,34(0,74-2,44)$ \\
\hline Mais que 20 horas & 29,9 & 1,00 & 1,00 \\
\hline
\end{tabular}

fissionais e condições sociais ${ }^{28}$. Investimento na aparência e em outros comportamentos como a seleção de comida é formatada pelas oportunidades sociais ao longo do tempo, estando relacionadas ao nível de riqueza. Assim, mudanças em circunstâncias sociais ou termos geracionais dentro de uma classe social podem alterar as prioridades relacionadas ao peso e à aparência, ou até mesmo mudar as normas sociais em relação ao modelo de corpo.
Também foi verificada associação da escolaridade do chefe de família com a insatisfação pelo excesso de peso. Os adolescentes cujos chefes de família apresentam o maior grau de escolaridade, o ensino superior completo, têm maior probabilidade de insatisfação pelo excesso. Na literatura pesquisada não foram encontrados estudos que utilizaram critérios e análises semelhantes (escolaridade do chefe de família e insatisfação com a imagem corporal), o que dificulta comparações 
Tabela 3. Prevalências e razões de prevalências utilizando-se como variável dependente a insatisfação pelo excesso de peso.

\begin{tabular}{|c|c|c|c|}
\hline Variáveis & $\%$ & $\begin{array}{l}\text { RP Bruta } \\
\text { (IC 95\%) }\end{array}$ & $\begin{array}{l}\text { RP Ajustada }{ }^{*} \\
\text { (IC 95\%) }\end{array}$ \\
\hline \multicolumn{4}{|l|}{ Sexo } \\
\hline Moças & 52,4 & $1,66(1,42-1,93)$ & $1,65(1,36-2,01)$ \\
\hline Rapazes & 31,6 & 1,00 & 1,00 \\
\hline \multicolumn{4}{|l|}{ Faixas etárias, anos } \\
\hline $13-14$ & 50,0 & $1,36(0,92-2,01)$ & $1,25(0,76-2,09)$ \\
\hline $15-16$ & 42,3 & $1,15(0,81-1,63)$ & $1,09(0,70-1,69)$ \\
\hline $17-18$ & 43,9 & $1,20(0,84-1,70)$ & $1,14(0,73-1,78)$ \\
\hline $19-21$ & 36,7 & 1,00 & 1,00 \\
\hline \multicolumn{4}{|l|}{ Classe econômica } \\
\hline Baixa & 29,3 & 1,00 & 1,00 \\
\hline Média & 44,3 & $1,51(1,01-2,29)$ & $1,55(0,94-2,53)$ \\
\hline Alta & 43,6 & $1,49(1,00-2,24)$ & $1,54(0,94-2,52)$ \\
\hline \multicolumn{4}{|l|}{ Renda } \\
\hline 1-3 salários mínimos & 42,7 & $1,02(0,86-1,20)$ & $0,96(0,77-1,20)$ \\
\hline 3,01-6 salários mínimos & 42,0 & 1,00 & 1,00 \\
\hline$>6$ salários mínimos & 48,3 & $1,15(0,93-1,41)$ & $1,14(0,86-1,51)$ \\
\hline \multicolumn{4}{|l|}{ Chefe da família } \\
\hline Analfabeto/3a Série Fund. & 38,3 & 1,00 & 1,00 \\
\hline Até $4^{\text {a }}$ Série Fundamental & 47,3 & $1,23(0,95-1,60)$ & $1,26(090-1,76)$ \\
\hline Fundamental completo & 37,3 & $0,97(0,73-1,28)$ & $1,04(0,73-1,48)$ \\
\hline Médio completo & 41,9 & $1,09(0,84-1,41)$ & $1,11(0,79-1,54)$ \\
\hline Superior completo & 50,3 & $1,31(1,01-1,69)$ & $1,38(1,08-1,77)$ \\
\hline \multicolumn{4}{|l|}{ Cor da pele } \\
\hline Branca & 42,9 & 1,00 & 1,00 \\
\hline Parda & 45,0 & $1,05(0,84-1,31)$ & $1,07(0,85-1,36)$ \\
\hline Preta & 44,9 & $1,05(0,88-1,24)$ & $1,12(0,83-1,51)$ \\
\hline \multicolumn{4}{|l|}{ Local de moradia } \\
\hline Periferia & 42,8 & $2,23(1,01-4,91)$ & $2,16(1,00-4,68)$ \\
\hline Centro & 49,5 & $2,58(1,14-5,80)$ & $2,43(1,10-5,35)$ \\
\hline Rural & 19,2 & 1,00 & 1,00 \\
\hline \multicolumn{4}{|l|}{ Trabalhadores } \\
\hline Não & 43,6 & $1,06(0,89-1,25)$ & $0,96(0,91-1,19)$ \\
\hline Sim & 41,3 & 1,00 & 1,00 \\
\hline \multicolumn{4}{|l|}{ Carga de trabalho semanal } \\
\hline Até 20 horas & 35,1 & 1,00 & 1,00 \\
\hline Mais que 20 horas & 41,7 & $1,19(0,74-1,89)$ & $1,26(0,69-2,29)$ \\
\hline
\end{tabular}

\%: frequência relativa, RP: razão de prevalência, IC95\%: intervalo de confiança de 95\%. " modelo ajustado por sexo e faixa etária.

bem como uma melhor compreensão deste fenômeno. Entretanto, pesquisas que investigaram, por exemplo, a escolaridade da mãe dos escolares e adolescentes participantes relatam achados divergentes. Enquanto Triches e Giugliani ${ }^{26}$ observaram que escolares com mães que possuíam menos de 8 anos de instrução estavam mais propensos à insatisfação com seu corpo, de modo geral, Finato et $\mathrm{al}^{11}$ não encontraram associação entre estas variáveis.
Algumas limitações importantes do presente estudo devem ser apontadas para que seja dada a devida atenção às mesmas em pesquisas futuras que tenham por objetivo investigar os fatores sociodemográficos que melhor expliquem a imagem corporal de adolescentes. Considerou-se apenas a escolaridade do chefe de família, podendo ser interessante, também, conhecer a ocupação profissional dos pais dos indivíduos participantes do estudo, informação relevante que 
reflete o nível econômico e a percepção simbólica destas famílias sobre corpo, beleza e valores em geral.

Sugere-se para estudos futuros, o conhecimento não somente da escolaridade, mas também da profissão dos familiares e o estado socioeconômico no momento da pesquisa em relação ao nível de mobilidade social que a família está vivendo. O controle desses aspectos poderá auxiliar a melhor compreensão de pesquisas que investiguem a nova e crescente classe econômica C no Brasil.

Em conclusão, os resultados encontrados no presente estudo permitem apontar que a insatis- fação com a imagem corporal pode ser explicada pelo sexo, grau de escolaridade do chefe da família, classe econômica, renda e local de moradia. Pensando nas possíveis consequências geradas pela insatisfação com o corpo, é de suma importância que reflexões sejam realizadas iniciando pelo ambiente escolar, com o intuito de orientar os estudantes sobre a necessidade de adoção e manutenção de hábitos de vida saudáveis, que incluem especialmente alimentação em quantidade e qualidade adequadas e a prática de atividades físicas, além da relação com o próprio corpo tendo em vista as mudanças ocorridas nesta fase da vida.

\section{Colaboradores}

Felden EPG trabalhou na elaboração do projeto, coleta e análise dos dados; GS Claumann atuou na análise e discussão dos dados e formatação do artigo; C Sacomori, LSE Daronco, FL Cardoso e A Pelegrini atuaram na discussão, interpretação e análise dos dados. 


\section{Referências}

1. Cash TF. Our first Body Image birthday: a year in review. Body Image 2005; 2(1):1-3.

2. Sacks O. Com uma perna só. São Paulo: Companhia das Letras; 2003.

3. Duchesne M, Mattos P, Fontenelle LF, Veiga H, Rizo L, Appolinario JC. Neuropsicologia dos transtornos alimentares: revisão sistemática da literatura. Rev Bras Psiquiatr 2004; 26(2):107-117.

4. Graup S, Pereira EF, Lopes AS, Araújo VC, Legnani RFS, Borgatto AF. Associação entre a percepção da imagem corporal e indicadores antropométricos de escolares. Rev Bras Educ Fís Esp 2008; 22(2):129-138.

5. Petroski EL, Pelegrini A, Glaner MF. Insatisfação corporal em adolescentes rurais e urbanos. Motri 2009; 5(4):13-25.

6. Pereira EF, Graup S, Lopes AS, Borgatto AF, Daronco LSE. Percepção da imagem corporal de crianças e adolescentes com diferentes níveis socio-econômicos na cidade de Florianópolis, Santa Catarina, Brasil. Rev Bras Saúde Matern Infant 2009; 9(3):253-262.

7. Castro IRR, Levy RB, Cardoso LO, Passos MD, Sardinha LMV, Tavares LF, Dutra SP, Martins A. Imagem corporal, estado nutricional e comportamento com relação ao peso entre adolescentes brasileiros. Cien Saude Colet 2010; 15(2):3099-3108.

8. Pereira EF, Teixeira CS, Gattiboni BD, Bevilacqua LA, Confortin SC, Silva TR. Percepção da imagem corporal e nível socioeconômico em adolescentes: revisão sistemática. Rev Paul Pediatr 2011; 29(3):423-429.

9. Dumith SC, Menezes AMB, Bielemann RM, Petresco S, Silva ICM, Linhares RS, Amorim TC, Duarte DV, Araújo CLP, Santos JV. Insatisfação corporal em adolescentes: um estudo de base populacional. Cien Saude Colet 2012; 17(9):2499-2505.

10. Fidelix YL, Minatto G, Ribeiro RR, Santos KD, Petroski EL. Dados sociodemográficos, estado nutricional e maturação sexual de escolares do sexo masculino: exposição à insatisfação com a imagem corporal. Rev Educ Fís/UEM 2013; 24(1):83-92.

11. Finato S, Rech RR, Migon P, Gavineski IC, Toni V, Halpern R. Insatisfação com a imagem corporal em escolares do sexto ano da rede municipal de Caxias do Sul, no Rio Grande do Sul. Rev Paul Pediatr 2013; 31(1):65-70.

12. Luiz RR, Magnanini MMF. A lógica da determinação do tamanho da amostra em investigações epidemiológicas. Cad Saúde Coletiva 2000; 8(2):9-28.

13. Stunkard AJ, Sorensen T, Schulsinger F. Use of the Danish adoption register for the study of obesity and thinnes. In: Ketty SS, Rowland LP, Sidman RL, Matthyse SW, editors. Genetics of Neurological and Psychiatric Disorders. New York: Raven; 1983. p. 115-120.

14. Associação Brasileira de Empresas de Pesquisa. Critério de Classificação Econômica Brasil. 2008. [acessado 2008 mar 5]. Disponível em: http://www.abep.org
15. Barros AJ, Hirakata VN. Alternatives for logistic regression in cross-sectional studies: an empirical comparison of models that directly estimate the prevalence ratio. BMC Med Res Methodol 2003; 3:21.

16. Glaner MF, Pelegrini A, Cordoba CO, Pozzobon ME. Associação entre insatisfação com a imagem corporal e indicadores antropométricos em adolescentes. Rev Bras Educ Fís Esporte 2013; 27(1):129-136.

17. Pelegrini A, Coqueiro RS, Beck CC, Ghedin KD, Lopes AS, Petroski EL. Insatisfação com a imagem corporal entre adolescentes estudantes: Associação com fatores sociodemográficos e estado nutricional. Cien Saude Colet 2014; 19(4):1201-1208.

18. Tiggemann M, Slater A. NetGirls: the internet, Facebook, and body image concern in adolescent girls. Int $J$ Eat Disord 2013; 46(6):630-633.

19. Clay D, Vignoles VL, Dittmar H. Body image and self-esteem among adolescent girls: testing the influence of sociocultural factors. J Res Adolesc 2005; 15(4): 451-477.

20. Passos MD, Gugelmin SA, Castro IRR, Carvalho MCVS. Representações sociais do corpo: um estudo com adolescentes do Município do Rio de Janeiro, Brasil. Cad Saude Publica 2013; 29(12):2383-2393.

21. Del Ciampo LA, Del Ciampo IRL. Adolescência e imagem corporal. Adolesc Saúde 2010; 7(4):55-59.

22. Nicholls D, Viner R. Eating disorders and weight problems. BMJ 2005; 330(7497):950-953.

23. Hunt TJ, Thienhaus O, Ellwood A. The mirror lies: body dysmorphic disorder. Am Fam Physician 2008; 78(2):217-222.

24. Azevedo AP, Ferreira AC, Silva PP, Caminha IO, Freitas CM. Dismorfia muscular: A busca pelo corpo hiper musculoso. Motri 2012; 8(1):53-66

25. Iriart JAB, Chaves JC, Orleans RG. Culto ao corpo e uso de anabolizantes entre praticantes de musculação. Cad Saude Publica 2009; 25(4):773-782.

26. Triches RM, Giugliani ERJ. Insatisfação corporal em escolares de dois municípios da região Sul do Brasil. Rev Nutr 2007; 20(2):119-128.

27. Bordo S. Unbearable weight. Los Angeles: University of California Press; 1993.

28. Bourdieu P. Distinction: A social critique of the judgement of taste. London: Routledge; 1984

Artigo apresentado em 05/06/2014

Aprovado em 10/06/2015

Versão final apresentada em 12/06/2015 
